\title{
Probing squared four-fermion operators of SMEFT with meson-mixing
}

\author{
Luiz Vale Silva ${ }^{a, *}$ \\ ${ }^{a}$ Instituto de Física Corpuscular, Universitat de València - Consejo Superior de Investigaciones Científicas, \\ Parc Científic, E-46980 Paterna, Valencia, Spain
}

E-mail: luizva@ific.uv.es

The Standard Model Effective Field Theory (SMEFT) is a universal way of probing New Physics (NP) manifesting as new heavy particle interactions with the Standard Model (SM) degrees of freedom, that respect the SM gauged symmetries. Higher order terms in the NP interactions possibly lead to sizable effects, mandatory for meaningful phenomenological studies, such as contributions to neutral meson-mixing, which typically pushes the scale of NP to energy scales much beyond the reach of direct searches in colliders. I discuss for the first time the leading-order renormalization of double-insertions of dimension-6 four-fermion operators that change quark flavor by one unit (i.e., $|\Delta F|=1, F=$ strange-, charm-, or bottom-flavor) by dimension- 8 operators relevant for meson-mixing (i.e., $|\Delta F|=2$ ) in SMEFT, and consider the phenomenological implications of contributions proportional to large Yukawas. Given the underlying interest of SMEFT to encode full-fledged models at low-energies, this work stresses the need of considering dimension-8 operators in phenomenological applications of dimension- 6 operators of SMEFT.

*** The European Physical Society Conference on High Energy Physics (EPS-HEP2021), ***

*** 26-30 July $2021 * * *$

*** Online conference, jointly organized by Universität Hamburg and the research center DESY ***

\footnotetext{
${ }^{*}$ Speaker
} 


\section{Introduction}

One strategy in searching for signs of New Physics (NP) - namely, phenomena that cannot be accommodated within the Standard Model (SM) - is the study of observables that are predicted by the SM to be suppressed, as for instance flavour changing neutral currents. A different strategy consists in looking for deviations in observables that are precisely predicted, such as the observables that contribute to the extraction of the elements of the Cabibbo-Kobayashi-Maskawa (CKM) matrix in the SM, among which meson-mixing observables play an important role [1].

Since the latter observables must involve, compared to the initial and final states and external momenta, the exchange of much heavier degrees of freedom (e.g., $W, Z$ bosons), an Effective Field Theory (EFT) provides at low energies a simpler picture of the underlying high-energy dynamics, in which Wilson coefficients and higher dimensional operators carry the fingerprints of such heavy particles. Similarly, EFTs can be used to investigate the effects of non-SM new heavy degrees of freedom (e.g., $\left.W^{\prime}, Z^{\prime}\right)$, that lead to new contact interactions among the SM degrees of freedom at low enough energies. The latter EFTs consist of higher dimensional operators suppressed by some new large scale $\Lambda_{\mathrm{NP}}$, typical of the NP extension, that encode in particular the flavour aspects of the new heavy sector, and their manifestation in observables that are suppressed in the SM or in observables that are precisely predicted can provide clear hints towards the discovery of NP.

The Standard Model Effective Field Theory (SMEFT) consists of the whole set of higher dimensional contact interactions that are consistent with Lorentz and the local symmetries of the $\mathrm{SM}$, and is particularly useful when a new weak interacting sector is considered, in which case it is meaningful to keep only the first terms of the power series in $\Lambda_{\mathrm{NP}}$. In the case of operators of dimension-6, the so-called Warsaw basis [2] is divided into eight categories, among which we have four-fermions, $\psi^{4}$ (see Eq. (2) below). Recently, explicit bases of dimension- 8 operators have been built $[3,4]$, among which one identifies operators involving four fermions, some of which can be thought of as composite dimension-2 Higgs-mass and dimension- 6 four-fermion operators, $H^{2} \times \psi^{4}$, or for instance "squared" dimension-4 Yukawa interactions, $\psi^{2} H \times \psi^{2} H$ (see Eq. (3) below).

In the case of NP effective operators involving fermion fields, effects that change flavour by one unity naturally lead to NP effects that change flavour by two units, which in the quark sector is efficiently probed by meson-mixing. This is going to be the main interest here, namely, the leading effect of double-insertions of dimension- 6 encoded in dimension- 8 operators, cf. Fig. 1; to spell out, the focus is on the renormalization of such double-insertions. Then, we will discuss phenomenological implications for one particular set of four-fermion operators.
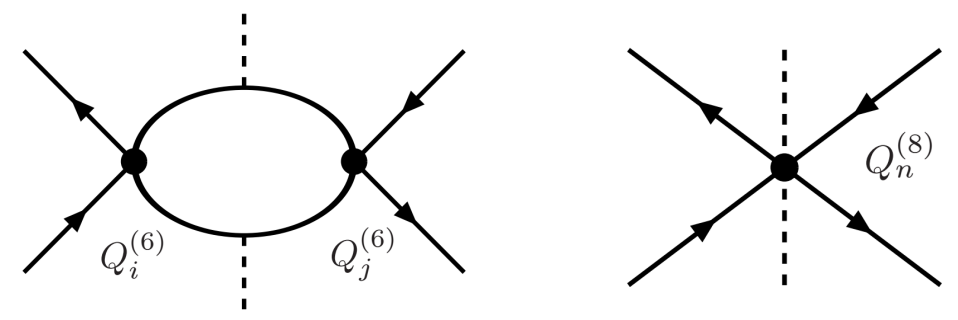

Figure 1: Double-insertion of dimension-6 $|\Delta F|=1$ operators (left), and tree-level single-insertion of dimension-8 $|\Delta F|=2$ operators (right). Filled circles represent the higher-dimensional effective interactions. 


\section{Effective operators in meson-mixing}

One illustration of the use of an EFT is provided by meson-mixing in the kaon sector in the SM [5-7]. Different flavours of the same type (here, up-type) can be combined as a result of the Glashow-Iliopoulos-Maiani (GIM) mechanism [8]. There are three sets of contributions that are qualitatively very different, according to the elements of the CKM matrix: boxes involving (1) topand up-, ${ }^{1}$ (2) charm- and up-, (3) charm-, top- and up-quarks. ${ }^{2}$ At the matching scale $\mu_{E W}$ (where $W, Z, H, t$ are integrated out and the first EFT is built from the full SM), case (1) is reproduced in the EFT by dim.-6 operators that change flavour by two units $(|\Delta F|=2)$, and cases (2) and (3) by dim. -6 operators that change flavour by one unity $(|\Delta F|=1)$, at the leading-order in the strong coupling. A (further) suppression due to the GIM mechanism is the elimination of large logarithmic contributions in the second case; at the same time, it makes double-insertions of dim.-6 operators finite in case (2), i.e., they do not require renormalization by dim.-8 operators. GIM does not operate in the same way in case (3), for which the main contribution is given by a large logarithm; simultaneously, GIM does not eliminate the divergence in double-insertions of dim.-6 operators, and case (3) requires renormalization by dim.-8 operators.

An analogous picture can be drawn in SMEFT. We consider a case analog to (3) above. First of all, we consider that the underlying NP sector does not generate contributions to dim.-6 $|\Delta F|=2$ operators, or at least that these contributions are suppressed. ${ }^{3}$ Then, we consider that a possible GIM-like mechanism in the NP sector does not eliminate the need to renormalize double-insertions of dim.-6 operators, i.e., large logarithms are present. Under these assumptions, the leading contribution to meson-mixing is captured by double-insertions of dim.-6 operators, that requires renormalization by dim. -8 operators.

To describe the resulting mixing of operators quantitatively, one must determine the anomalous dimension tensor $\gamma_{i j, n}$ : given a set of Green's functions with two insertions of dim.-6 operators (indexed by $i, j$ ) one calculates the counter-terms proportional to dim.-8 operators (indexed by $n$ ), needed to renormalize the divergences resulting from the double-insertions. Large logarithms are resummed via the Renormalization Group (RG) evolution:

$$
\mu \frac{d}{d \mu} C_{n}^{(8)}(\mu)=\Sigma_{i, j} C_{i}^{(6)}(\mu) C_{j}^{(6)}(\mu) \gamma_{i j, n}+\Sigma_{m} C_{m}^{(8)}(\mu) \gamma_{m n}
$$

where the superscripts of the Wilson coefficients give the dimension of the corresponding operator. Solving these RG equations, the term proportional to the two dim.- 6 Wilson coefficients, $C_{i}^{(6)}\left(\Lambda_{\mathrm{NP}}\right)$ times $C_{j}^{(6)}\left(\Lambda_{\mathrm{NP}}\right)$ (assumed non-zero), leads to the logarithm $\log \left(\Lambda_{\mathrm{NP}} / \mu_{E W}\right)$, analog to the logarithmic enhancement of case (3) above; also under the assumptions of the previous paragraph, the values of the dim.-8 Wilson coefficients $C_{n}^{(8)}\left(\Lambda_{\mathrm{NP}}\right)$ are sub-leading (in the strong coupling).

For simplicity, we focus on double-insertions of the same operator, with the same flavour content; furthermore, we focus on double-insertions of four-fermion operators. Although here we focus on meson-mixing, a similar discussion would hold for rare decays.

\footnotetext{
${ }^{1}$ Case (1) is the dominant contribution to neutral- $B_{(s)}$ meson-mixing in the SM.

${ }^{2}$ The same qualitative discussion holds for the different grouping of contributions considered in [9].

${ }^{3}$ For instance, in Left-Right Models the large masses of the extended (neutral) scalar sector suppress their tree-level contributions to meson-mixing, see e.g. [10].
} 


\begin{tabular}{|c|c|c|c|c|}
\hline & flavours & $B(f=b, i=d)$ & $B_{s}(f=b, i=s)$ & $K(f=s, i=d)$ \\
\hline \multirow{2}{*}{$\begin{array}{l}\frac{\vec{\omega}}{\infty 0} \\
\cdot \stackrel{\oplus}{\omega}\end{array}$} & $33 f i,(33 i f)^{\dagger}$ & $\left|\left(\tilde{C}_{q u q d}^{(1)}\right)^{2}\right|<\frac{1}{(7.5 \mathrm{TeV})^{4}}$ & $\left|\left(\tilde{C}_{q u q d}^{(1)}\right)^{2}\right|<\frac{1}{(4.1 \mathrm{TeV})^{4}}$ & $\left|\operatorname{Im}\left\{\left(\tilde{C}_{q u q d}^{(1)}\right)^{2}\right\}\right|<\frac{1}{(58 \mathrm{TeV})^{4}}$ \\
\hline & $f 33 i,(i 33 f)^{\dagger}$ & $\left|\left(\tilde{C}_{q u q d}^{(1)}\right)^{2}\right|<\frac{1}{(4.8 \mathrm{TeV})^{4}}$ & $\left|\left(\tilde{C}_{q u q d}^{(1)}\right)^{2}\right|<\frac{1}{(2.6 \mathrm{TeV})^{4}}$ & $\left|\operatorname{Im}\left\{\left(\tilde{C}_{\text {quqd }}^{(1)}\right)^{2}\right\}\right|<\frac{1}{(37 \mathrm{TeV})^{4}}$ \\
\hline \multirow{2}{*}{$\frac{\vec{d}}{0}$} & $33 f i,(33 i f)^{\dagger}$ & $\left|\left(\tilde{C}_{q u q d}^{(8)}\right)^{2}\right|<\frac{1}{(6.1 \mathrm{TeV})^{4}}$ & $\left|\left(\tilde{C}_{q u q d}^{(8)}\right)^{2}\right|<\frac{1}{(3.4 \mathrm{TeV})^{4}}$ & $\left|\operatorname{Im}\left\{\left(\tilde{C}_{q u q d}^{(8)}\right)^{2}\right\}\right|<\frac{1}{(47 \mathrm{TeV})^{4}}$ \\
\hline & $f 33 i,(i 33 f)^{\dagger}$ & $\left|\left(\tilde{C}_{q u q d}^{(8)}\right)^{2}\right|<\frac{1}{(6.1 \mathrm{TeV})^{4}}$ & $\left|\left(\tilde{C}_{q u q d}^{(8)}\right)^{2}\right|<\frac{1}{(3.4 \mathrm{TeV})^{4}}$ & $\left|\operatorname{Im}\left\{\left(\tilde{C}_{q u q d}^{(8)}\right)^{2}\right\}\right|<\frac{1}{(47 \mathrm{TeV})^{4}}$ \\
\hline
\end{tabular}

Table 1: Bounds on the Wilson coefficients of the operators in Eq. (2). The first column indicates the flavour indices (bounds correspond to the combination of the two cases provided, with no interference). The three remaining columns give bounds accordingly to the meson system. Limits are $95 \%$ Confidence Level bounds.

\subsection{Example}

We discuss a specific example, corresponding to the following two operators:

$$
\text { dim.-6: } \quad Q_{\text {quqd }}^{\text {(singlet) }}=\left(\bar{q}^{m} u\right) \epsilon_{m n}\left(\bar{q}^{n} d\right), \quad Q_{q u q d}^{\text {(octet) }}=\left(\bar{q}^{m} T^{A} u\right) \epsilon_{m n}\left(\bar{q}^{n} T^{A} d\right)
$$

where $u, d$ are right-handed quark fields of respectively up- and down-type flavours, and $q$ are left-handed quark fields; flavour and color indices are omitted, ${ }^{4}$ while weak-isospin indices are indicated by $m, n$. With these two operators, we have contributions to meson-mixing as shown in Fig. 1 (left) in which we consider that the internal fermions running inside the loop are top-quarks. The resulting effect is then proportional to large Yukawas. There is also some enhancement from the color-group structure of these diagrams. The relevant dim.-8 operators are the following ones:

$$
\text { dim.-8: } \quad Q_{q^{2} d^{2} H^{2}}^{\text {(singet) }}=(\bar{q} d H)(\bar{q} d H), \quad Q_{q^{2} d^{2} H^{2}}^{\text {(otet }}=\left(\bar{q} T^{A} d H\right)\left(\bar{q} T^{A} d H\right)
$$

which are required to renormaize double-insertions of the operators in Eq. (2).

Since here the internal flavour is a top, below the EW scale the relevant operators are:

$$
\text { low-energies: } \quad \tilde{O}_{2}^{\psi \xi}=\left(\bar{\psi}_{L}^{\alpha} \xi_{R}^{\alpha}\right)\left(\bar{\psi}_{L}^{\beta} \xi_{R}^{\beta}\right), \quad \tilde{O}_{3}^{\psi \xi}=\left(\bar{\psi}_{L}^{\alpha} \xi_{R}^{\beta}\right)\left(\bar{\psi}_{L}^{\beta} \xi_{R}^{\alpha}\right)
$$

where $\alpha, \beta$ are color indices, and flavours are $\psi, \xi=b, s, d$. Their hadronic matrix elements are calculated in [11,12], which are chirally enhanced in the case of kaons.

Finally, one sets constraints on the NP Wilson coefficients of the operators in Eq. (2) based on their contributions to different meson-mixing observables. We exploit the bounds provided in $[13,14]$ on generic NP contributions. Despite being an effect coming from a dim.-8 operator and being generated at one-loop, given the previous enhancements and the precision with which many of these observables are known, one reaches a sensitivity to multi-TeV NP effects, as shown in Tab. 1 and Fig. 2, for both singlet and octet structures. A certain number of comments is in order:

- Sub-leading effects above the EW scale may be numerically relevant if the leading logarithm is not largely dominant; their determination, however, is beyond the scope of this work.

- Short-distance QCD effects below the EW scale have been included [15].

\footnotetext{
${ }^{4}$ The matrices $T^{A}$ are normalised as $\operatorname{tr}\left\{T^{A} T^{B}\right\}=2 \delta^{A B}$.
} 


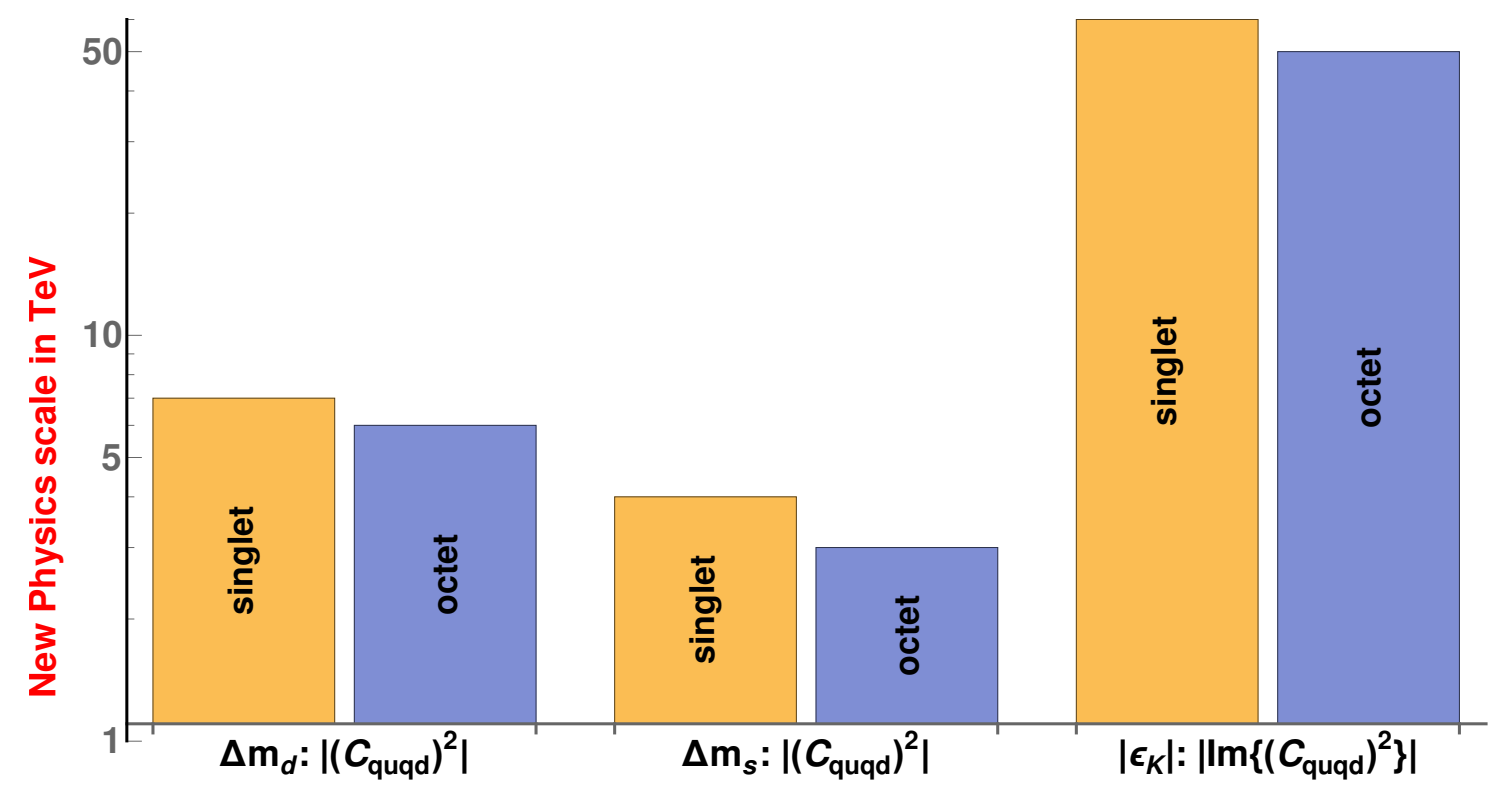

Figure 2: Bounds on the NP Wilson coefficients of the contact interactions in Eq. (2). The vertical axis corresponds to the typical scale found in Tab. 1. This plot shows sensitivity to multi-TeV NP effects.

- Other observables can also constrain the same NP effective couplings; e.g., many other operators are radiatively generated through single-insertions [16-18]; it has been explicitly verified that these do not lead to the main phenomenological bounds in the present example.

The anomalous dimension tensor for the present example together with those for an enlarged set of operators will be provided in a future publication. Other than top-loops, one can also have other internal heavy flavours, namely, charm- and bottom-quarks, and tau-leptons (much heavier than kaons). In these cases double-insertions have also to be considered below the EW scale, with a different set of anomalous dimension tensor elements. A further complication is that other dim.-8 operators can also show up, schematically: $\psi^{4} H D, \psi^{4} D^{2}$, and $\psi^{4} X$. Their contributions are however suppressed by the external masses (or the external momentum scale) and sub-leading if the internal flavour is heavy enough.

\section{Conclusions}

I have discussed effects from a generic heavy NP sector that are encoded in higher dimensional operators. More exactly, I have discussed the renormalization by dim.-8 operators of doubleinsertions of dim.- 6 operators, where the latter changes flavour number by one unity. At energy scales much below the characteristic scale of NP, the effects of double-insertions are constrained by meson-mixing observables, which receive suppressed contributions from the SM that are precisely predicted in many cases. Given the level of experimental accuracy reached for these observables, even loop-suppressed double-insertions lead to meaningful and powerful bounds on NP, probing energy scales much beyond the direct reach of colliders. 


\section{References}

[1] J. Charles et al. SM CKM fit and constr. on $\Delta F=2$ NP. Phys. Rev. D, 91(7):073007, 2015.

[2] B. Grzadkowski, M. Iskrzynski, M. Misiak, and J. Rosiek. Dimension-Six Terms in the Standard Model Lagrangian. JHEP, 10:085, 2010.

[3] Christopher W. Murphy. Dimension-8 operators in the SMEFT. JHEP, 10:174, 2020.

[4] Hao-Lin Li, Zhe Ren, Jing Shu, Ming-Lei Xiao, Jiang-Hao Yu, and Yu-Hui Zheng. Complete set of dimension-eight operators in the SMEFT. Phys. Rev. D, 104(1):015026, 2021.

[5] F. J. Gilman and M. B. Wise. $K^{0} \bar{K}^{0}$ Mix. in the 6 Quark Model. Phys. Rev. D, 27:1128, 1983.

[6] Stefan Herrlich and Ulrich Nierste. Enhancement of the $K(L)-K(S)$ mass difference by short distance QCD corrections beyond leading logarithms. Nucl. Phys. B, 419:292-322, 1994.

[7] S. Herrlich and U. Nierste. The Complete $|\Delta S|=2$ - Hamiltonian in the NLO. Nucl. Phys. B, 476:27-88, 1996.

[8] S. L. Glashow, J. Iliopoulos, and L. Maiani. Weak Interactions with Lepton-Hadron Symmetry. Phys. Rev. D, 2:1285-1292, 1970.

[9] Joachim Brod, Martin Gorbahn, and Emmanuel Stamou. Standard-Model Prediction of $\epsilon_{K}$ with Manifest Quark-Mixing Unitarity. Phys. Rev. Lett., 125(17):171803, 2020.

[10] Véronique Bernard, Sébastien Descotes-Genon, and Luiz Vale Silva. Short-distance QCD corrections to $K^{0} \bar{K}^{0}$ mixing at NLO in Left-Right models. JHEP, 08:128, 2016.

[11] N. Carrasco et al. $\Delta \mathrm{S}=2$ and $\Delta \mathrm{C}=2$ bag parameters in the standard model and beyond from $\mathrm{N}_{f}=2+1+1$ twisted-mass lattice QCD. Phys. Rev. D, 92(3):034516, 2015.

[12] A. Bazavov et al. $B_{(s)}^{0}$-mixing matrix elements from lattice QCD for the Standard Model and beyond. Phys. Rev. D, 93(11):113016, 2016.

[13] J. Charles, S. Descotes-Genon, Z. Ligeti, S. Monteil, M. Papucci, and K. Trabelsi. Future sensitivity to new physics in $B_{d}, B_{s}$, and K mixings. Phys. Rev. D, 89(3):033016, 2014.

[14] J. Charles, S. Descotes-Genon, Z. Ligeti, S. Monteil, M. Papucci, K. Trabelsi, and L. Vale Silva. NP in $B$ meson mixing: future sensitivity and limitations. Phys. Rev. D, 102(5):056023, 2020.

[15] Andrzej J. Buras, Sebastian Jager, and Jorg Urban. Master formulae for Delta F=2 NLO QCD factors in the standard model and beyond. Nucl. Phys. B, 605:600-624, 2001.

[16] Elizabeth E. Jenkins, Aneesh V. Manohar, and Michael Trott. Ren. Group Evolution of the SM Dim. Six Operators I: Formalism and lambda Dependence. JHEP, 10:087, 2013.

[17] Elizabeth E. Jenkins, Aneesh V. Manohar, and Michael Trott. Ren. Group Evolution of the SM Dim. Six Operators II: Yukawa Dependence. JHEP, 01:035, 2014.

[18] R. Alonso, E. E. Jenkins, A. V. Manohar, and M. Trott. Ren. Group Evolution of the SM Dim. Six Operators III: Gauge Coupling Dependence and Pheno. JHEP, 04:159, 2014. 